NASA Contractor Report 195379

AIAA-94-3141

\title{
Stationary Plasma Thruster Ion Velocity Distribution
}

David H. Manzella

NYMA, Inc.

Engineering Services Division

Brook Park, Ohio

August 1994

Prepared for

Lewis Research Center

Under Contract NAS3-27186

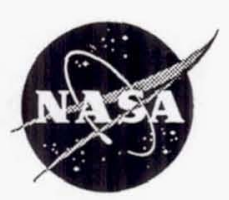

National Aeronautics and

Space Administration 


\title{
Stationary Plasma Thruster Ion Velocity Distribution
}

\author{
David H. Manzella ${ }^{\dagger}$ \\ NYMA, Inc. \\ Engineering Services Division \\ Brook Park, Ohio 44142
}

A non-intrusive velocity diagnostic based on laser induced fluorescence of the $5 d^{4} F_{5 / 2}-6 p^{4} D_{5 / 2}$ singly ionized xenon transition was used to interrogate the exhaust of a $1.5 \mathrm{~kW}$ Stationary Plasma Thruster (SPT). A detailed map of plume velocity vectors was obtained using a simplified, cost-effective, non-intrusive, semiconductor laser based scheme. Circumferential velocities on the order of $250 \mathrm{~m} / \mathrm{s}$ were measured which implied induced momentum torques of approximately $5 \times 10^{-2} \mathrm{~N}-\mathrm{cm}$. Axial and radial velocities were evaluated one $\mathrm{mm}$ downstream of the cathode at several locations across the width of the annular acceleration channel. Radial velocities varied linearly with radial distance. A maximum radial velocity of $7500 \mathrm{~m} / \mathrm{s}$ was measured $8 \mathrm{~mm}$ from the center of the channel. Axial velocities as large as $16,500 \mathrm{~m} / \mathrm{s}$ were measured.

\section{Nomenclature}

A fine structure constant accounting for nuclear magnetic moment, $\mathrm{MHz}$

$B$ fine structure constant accounting for electric quadrupole interaction, $\mathrm{MHz}$

C fine structure constant, $\mathrm{MHz}$

c speed of light, $\mathrm{m} / \mathrm{s}$

$F$ total angular momentum quantum number

I nuclear spin quantum number

J total electronic angular momentum quantum number

$k$ Boltzmann's constant, $\mathrm{J} / \mathrm{K}$

$m$ mass of ion, $\mathrm{kg}$

$T$ temperature, $\mathrm{K}$

$v_{i}$ ion velocity in direction of laser, $\mathrm{m} / \mathrm{s}$

$W_{F}$ quantum state term energy including hyperfine splitting, $\mathrm{MHz}$

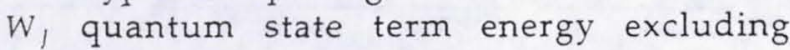
hyperfine splitting, $\mathrm{MHz}$

$\Delta v$ Doppler shift, $\mathrm{cm}^{-1}$

$\Delta v_{D}$ Doppler width (FWHM), $\mathrm{cm}^{-1}$

$v_{0}$ unshifted frequency of electronic transition, $\mathrm{cm}^{-1}$

$\kappa$ constant

\section{Introduction}

The suitability of ion accelerators with crossed electric and magnetic fields, such as the Russian stationary plasma thruster (SPT), for satisfying various propulsion mission requirements is currently being assessed. Spacecraft integration issues are a major consideration for such a system to be successfully employed on next-generation spacecraft. NASA has taken an active role in assessing integration issues pertinent to the SPT using a thruster provided by the Ballistic Missile Defense
Organization (BMDO)..$^{1-5}$ One specific issue relevant to spacecraft integration of a SPT is the possible momentum torques generated by circumferential ion motion. Sputtering of spacecraft surfaces by ion bombardment also needs to be evaluated.

The direction and magnitude of ion velocity vectors can be used to perform this type of spacecraft integration assessment. Such data would also aid in a detailed performance assessment because the spatial integration of the density weighted velocity field determines the thrust vector. For example, the measurement of velocity in directions other than the direction of the thrust permits an evaluation of thrust inefficiencies due to beam divergence. Additionally, a detailed understanding of the flow field in the region of critical engine surfaces can provide information on sputtering due to impingment.

This paper describes the direct measurement of the ion velocity at the exit of a SPT based on a high-spatial-resolution, non-intrusive optical diagnostic. To date the use of non-intrusive optical diagnostics applied to SPT's consisted of emission spectroscopy measurements of excited state populations in the exit plane of the SPT$100 .{ }^{5}$ In this investigation the optical technique employed to measure ion velocities was laser induced fluorescence (LIF) using a semiconductor laser. LIF is particularly well suited for measurements near the thruster exit where survivability of intrusive probes is an issue and where steep property gradients make high spatial resolution advantageous. Measurements of the velocity field at the thruster exit are 
desirable because possible facility interactions are minimized at this location. These results can then be extrapolated to determine the ion impacts at various distances from the thruster. The use of a semiconductor laser enables implementation of a such a diagnostic for a fraction of the cost of traditional systems.

\section{Theory}

Velocity measurement using laser induced fluorescence is based on the Doppler effect. Laser induced fluorescence can be described as two separate processes: photon absorption and subsequent spontaneous emission. Absorption occurs when the interaction between an incident photon and an ion results in the promotion of a bound electron from one energy quantum state to a higher energy quantum state. This may take place when the photon has the same energy as the electronic transition. The excited state is not stable and will spontaneously decay while emitting a photon in an arbitrary direction.

If the ion is moving relative to the laser beam, the laser photon energy in the moving frame of reference is Doppler shifted by an amount proportional to the relative velocity between the ion and laser. The change in photon energy (or laser frequency) needed to be resonant with the electronic transition of a moving ion is referred to as a Doppler shift and can be related to the resonant frequency for a stationary ion, the component of the ion's velocity in the direction of the laser beam, and the speed of light according to:

$$
\Delta v=v_{0} \cdot\left(v_{i} / c\right)
$$

This velocity diagnostic concept has successfully been implemented by several investigators in high velocity plasma flows. . $^{6}$

In this investigation a non-resonant scheme was implemented (ie. spontaneous decay of the upper state to a state other than the lower laser coupled state). The optically allowed $5 \mathrm{~d}^{4} \mathrm{~F}_{5 / 2}$ $6 \mathrm{p}^{4} \mathrm{D}_{5 / 2}$ transition of singly ionized xenon which has an energy of $11976.72 \mathrm{~cm}^{-1}(834.7 \mathrm{~nm})$ was stimulated while monitoring spontaneous emission from the $6 p^{4} D_{5 / 2}-6 s^{4} P_{3 / 2}$ transition at $18447.95 \mathrm{~cm}^{-1}(541.9 \mathrm{~nm})$. The laser coupled transition at $11976.72 \mathrm{~cm}^{-1}$ was chosen for convenience as it was easily accessible using a semiconductor laser.

Additional information such as kinetic ion temperatures and magnetic field strengths may be determined from fluorescence excitation line shapes. This type of analysis requires a detailed line shape model which should include isotopic effects, hyperfine splitting, Zeeman splitting, saturation broadening due to excessive laser power, and other broadening mechanisms associated with the plasma such as Doppler broadening, resonance broadening, Stark broadening, lifetime broadening, and transit time broadening. For the conditions in the SPT plume, Doppler broadening should dominate, so a model incorporating isotopic effects, hyperfine splitting, and Doppler broadening was constructed.

Naturally occurring xenon is composed of seven isotopes. For each of these isotopes the energy of the measured transition is slightly shifted. The magnitude of the isotopic shift can be determined based on the change in the center of gravity of the ion, the change in the nuclear radius of the ion, and the degree of interaction between the ion's electrons. The change in the center of gravity can accurately be calculated. Calculations of the other effects requires accurate wave functions presently not available for xenon. Because of this, experimentally determined isotope shift values were sought, though they were not available for the investigated transition. In the absence of these data, published data for a similar transition, the $5 \mathrm{~d}$ ${ }^{4} \mathrm{D}_{7 / 2}-6 \mathrm{p}^{4} \mathrm{P}_{5 / 2}$ transition at $16521.24 \mathrm{~cm}^{-1}(605.1$ $\mathrm{nm})$ were used.

Table I: Isotope Shifts

\begin{tabular}{ccc}
\hline \hline Isotope & $\begin{array}{c}\text { \% natural } \\
\text { abundance }\end{array}$ & $\begin{array}{c}\text { Isotope Shift } \\
\text { (rel to } \mathrm{Xe}^{132} \text { ) } \mathrm{MHz}^{10}\end{array}$ \\
\hline $\mathrm{Xe}^{132}$ & 26.89 & 0 \\
$\mathrm{Xe}^{129}$ & 26.44 & 162 \\
$\mathrm{Xe}^{131}$ & 21.18 & 99 \\
$\mathrm{Xe}^{134}$ & 10.44 & 80 \\
$\mathrm{Xe}^{136}$ & 8.87 & 12 \\
$\mathrm{Xe}^{130}$ & 4.08 & -82 \\
$\mathrm{Xe}^{128}$ & 1.92 & -145 \\
\hline \hline
\end{tabular}

Hyperfine splitting occurring in the odd numbered isotopes gives rise to additional structure of the excitation line shape. $\mathrm{Xe}^{129}$ has a nuclear spin quantum number $I$ of $1 / 2$ which splits the upper and lower states into two states. This results in four transitions. $\mathrm{Xe}^{131}$ has a nuclear spin quantum number of $3 / 2$ which splits both the upper and lower states into four individual states. Transitions with $\Delta F= \pm 1,0$ are allowed, except from $F=0$ to 0 . The total angular momentum quantum number $F$ takes values from:

$$
F=I+J, I+J-1, \ldots \ldots,|I-J|
$$


where $J$ is the total angular momentum of the electrons. This results in ten allowed transitions which are shown in Fig. 1.

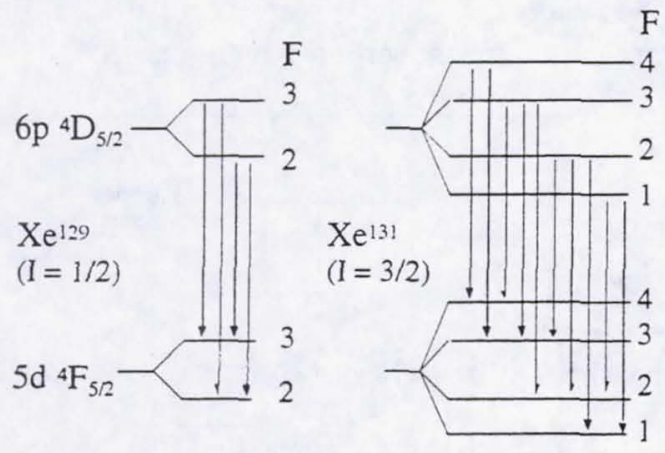

Fig. 1 Hyperfine Levels and Transitions for $\mathrm{Xe}^{129}$ and $\mathrm{Xe}^{131}$

The energy of the individual hyperfine levels is determined by modifying the term energy for the individual odd isotopes with correction terms due to the magnetic moment of the nucleus and the electric quadrupole coupling between the nucleus and electrons:

$$
W_{F}=W_{J}+A C / 2+B \frac{3 / 4 C(C+1)-I(I+1) J(J+1)}{2 I(2 I-1) J(2 J-1)}
$$

where $W_{F}$ is the term energy including fine structure and $W_{J}$ is the term energy accounting for everything other than hyperfine splitting. $A$ is a fine structure constant which corrects for the nuclear magnetic moment and $B$ is a fine structure constant that accounts for the electric quadrupole interaction between the nucleus and electrons. The quadrupole interaction vanishes for $\mathrm{Xe}^{129}$ which has a spherical nuclear charge distribution. The constant $C$ can be calculated based on quantum mechanical considerations.

$$
C=F(F+1)-I(I+1)-J(J+1)
$$

Typically fine structure constants are determined experimentally. These constants were unavailable for the transition considered in this investigation.

As a result of these isotopic shifts and hyperfine splitting the $5 d^{4} F_{5 / 2}-6 p^{4} D_{5 / 2}$ transition is split into 20 individual transitions. The relative intensities of the contribution from each isotope is determined based on the relative abundance of each isotope. The relative intensity of each hyperfine component can be calculated based on quantum mechanical considerations. For this transition $(J=5 / 2$ to $J=5 / 2)$ if $\Delta F=0$

$$
I=\frac{\kappa[J(I+1)+F(F+1)-I(I+1)]^{2}(2 F+1)}{F(F+1)}
$$

if $\Delta F=1$

$$
I=\frac{-K(J+F+I+1)(J+F-I)(J-F+I+1)(J-F-I)}{F}
$$

and if $\Delta F=-1$

$$
I=\frac{-\kappa(J+F+I+2)(J+F-I+1)(J-F+1)(J-F-I-1)}{(F+1)}
$$

where $\kappa$ is an unknown constant.

The width of each of the 20 individual components of the transition manifold may have been affected by any of the broadening phenomena previously mentioned. Additionally, each state may have been Zeeman split into $2 F+1$ states if magnetic field effects are substantial. For this model only Doppler broadening was considered. Doppler broadening is the result of the spread in velocity for the species being investigated. This spread in velocity classes is generally attributed to the random thermal motion of the ions. This gives rise to a Gaussian shape for each of the transitions with a half width of:

$$
\Delta v_{D}=v_{0} \sqrt{\frac{8 k T \ln 2}{m c^{2}}}
$$

\section{Apparatus and Procedure}

The investigation of ion velocity in the exhaust of the SPT-100 was conducted in a vacuum chamber measuring $1.5 \mathrm{~m}$ in diameter by 5 meters in length. The pumping for this chamber consisted of four $0.82 \mathrm{~m}$ oil diffusion pumps (each rated at $30,000 \mathrm{l} / \mathrm{s}$ ), a lobe type blower, and two piston type roughing pumps. The SPT was mounted in a $60 \mathrm{~cm}$ diameter spool piece separated from the main chamber with a $60 \mathrm{~cm}$ isolation valve. The smaller diameter of the spool piece permitted the entire optical system to be located outside the vacuum facility. The locations of the spool piece relative to the tank, the thruster relative to the spool piece, and the diffusion pumps are shown in Fig. 2.

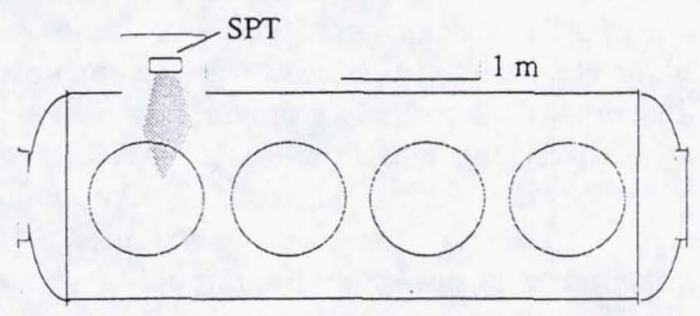

Fig. 2 Vacuum Chamber with SPT 
The SPT-100 used for this investigation was fabricated by Fakel Enterprises in Russia and was provided by BMDO. It was previously used in several experimental investigations and a more complete description is given elsewhere. ${ }^{1} \mathrm{~A}$ photograph of this thruster is shown in Fig. 3. The outer diameter of the annular discharge

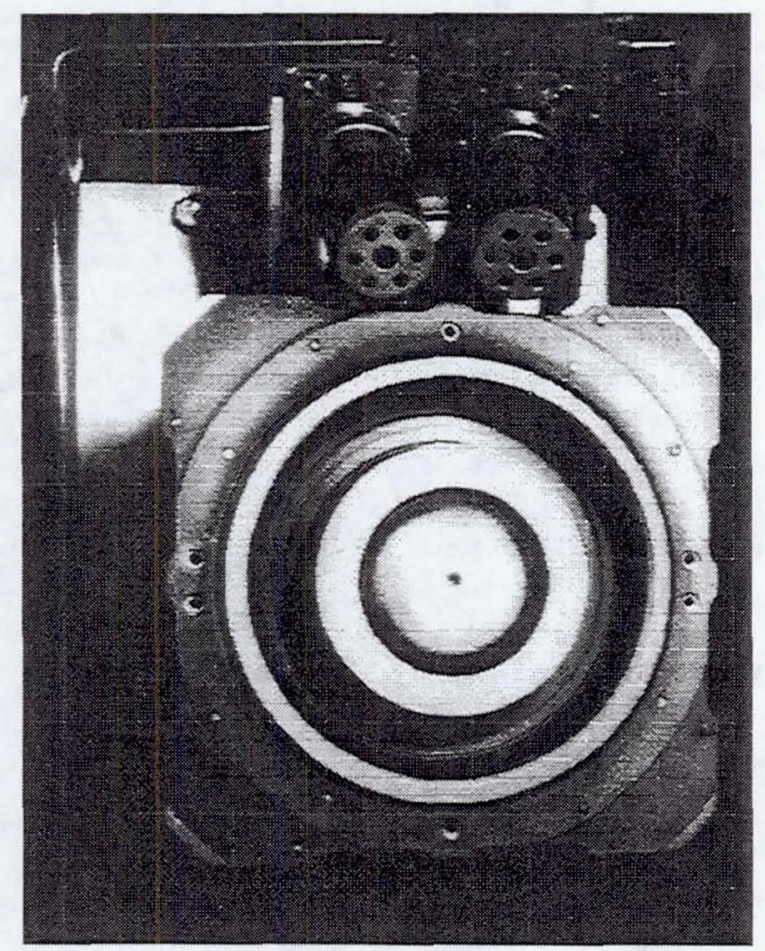

Fig. 3 SPT-100

chamber was $10 \mathrm{~cm}$. The inner diameter was 5.6 $\mathrm{cm}$. The exit plane of the cathode/neutralizer assembly was $1 \mathrm{~cm}$ downstream of the thruster exit plane. The thruster was run on commercially available research grade xenon ( $99.9995 \%$ pure) at a nominal operating condition of $300 \mathrm{~V}$ discharge voltage, $4.5 \mathrm{~A}$ discharge current, and 5 $\mathrm{mg} / \mathrm{s}$ propellant flow. The total gas flow was internally divided between the anode and cathode. The flow system used to supply the gas to the thruster consisted of a needle valve and flow meter downstream of a pressure regulator, and a 250 liter compressed xenon cylinder. The flow meter was calibrated using a volume displacement method. The power supply used to run the discharge was a $600 \mathrm{~V}, 25 \mathrm{~A} \mathrm{DC}$ power supply operated with an external $10 \mathrm{Ohm}$ ballast resistor. A $100 \mathrm{mF}$ capacitor was installed across the discharge to minimize the impact of plasma oscillations on the power supply. Additional DC power supplies were used to run the heater cathode during start up and the keeper prior to initiation of the main discharge. A simplified schematic of the electrical configuration and the instrumentation used to monitor operation is shown in Fig. 4. In all cases the thruster was allowed to reach steady state conditions before spectroscopic measurements were taken.

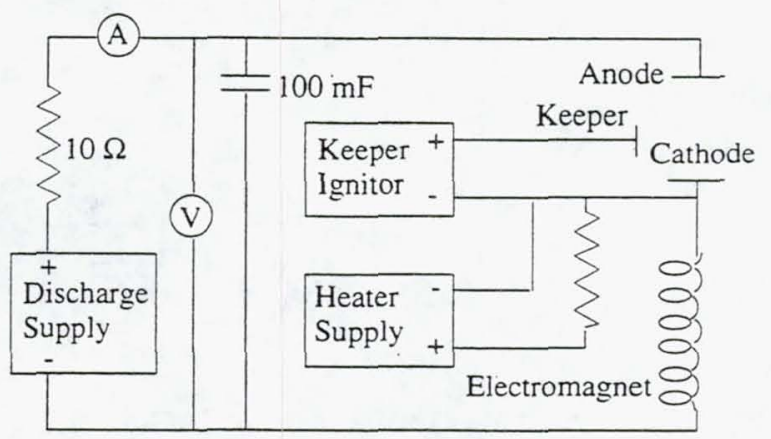

Fig. 4 SPT Electrical Schematic

The experimental apparatus for the laser induced fluorescence measurements consisted of the laser system, associated positioning and focusing optics, and the fluorescence detection system. The laser used was a commercially available continuous wave, single-mode, $100 \mathrm{~mW}$ GaAlAs semiconductor laser with a nominal center wavelength of $845 \mathrm{~nm}$ and a line width of approximately $15 \mathrm{MHz}\left(0.0005 \mathrm{~cm}^{-1}\right)$. Frequency stability of the laser was optimized by using a very stable, low noise battery-powered current source. Laser temperature was actively regulated using a thermoelectric heater/cooler. Frequency tuning was accomplished by varying the injection current.

During a typical scan the laser frequency was monitored using an evacuated scanning Michelson interferometer with a resolution of $0.01 \mathrm{~cm}^{-1}$. The mode structure of the laser was evaluated with a 2 Ghz free spectral range confocal etalon. A anamorphic prism pair was used to circularize the elliptical beam and a Faraday isolator was used to minimize optical feedback to the laser cavity. The output laser power was monitored using a calibrated power meter.

The laser beam was directed to the appropriate optical access port on the spool piece using front surface mirrors. A $400 \mathrm{~mm}$ focal length lens was used to focus the laser to a submillimeter diameter at the probe volume. Nonresonant fluorescence at $541.9 \mathrm{~nm}$ was collected using a $400 \mathrm{~mm}$ focal length lens aligned orthogonally to the laser beam. A fixed wavelength $0.5 \mathrm{~m}$ Czerny-Turner monochromator was used as a spectral filter. The fluorescence was converted to a signal by a bialkali type 
photo multiplier. The current produced by the photo multiplier tube was monitored as a voltage across a $1 \mathrm{k} \Omega$ load resistor. This voltage was measured using a digital lock-in-amplifier. The lock-in amplifier permitted phase sensitive detection of the fluorescence signal at $1.2 \mathrm{kHz}$, the frequency the laser beam was modulated at using a mechanical chopper. Phase sensitive detection was used to discriminate spontaneous emission at $541.9 \mathrm{~nm}$ from the non-resonant fluorescence signal at the same frequency. The entire laser induced fluorescence apparatus is shown schematically in Fig. 5.

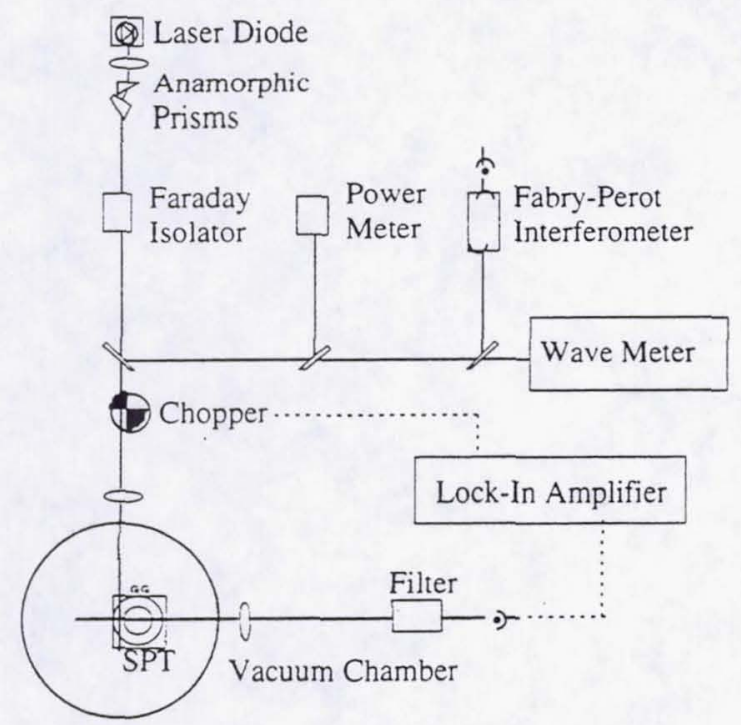

Fig. 5 Optical Schematic (for circumferential velocity determination)

A series of three different tests were conducted to investigate ion velocities in the axial, radial, and circumferential directions. These different velocity components were measured by bringing the laser into the probe volume in three different directions. The circumferential velocity was measured at the exit of the discharge chamber in the center of the annular channel with the beam tangent to the annulus as depicted in Fig. 4. The radial and axial velocity components were measured at various positions at a location $11 \mathrm{~mm}$ downstream of the discharge chamber exit plane. The thruster was moved laterally with a computer-controlled, stepper-motor-driven, linear actuator system to accomplish this. The locations of these measurements are shown in Fig. 6. The radial velocity component was measured by directing the laser in the minus $r$ direction (also shown in Fig. 6). The axial velocity component was determined in conjunction with radial velocity by bringing the laser into the probe volume at an angle of 60 degrees relative to the axial direction in a horizontal plane.

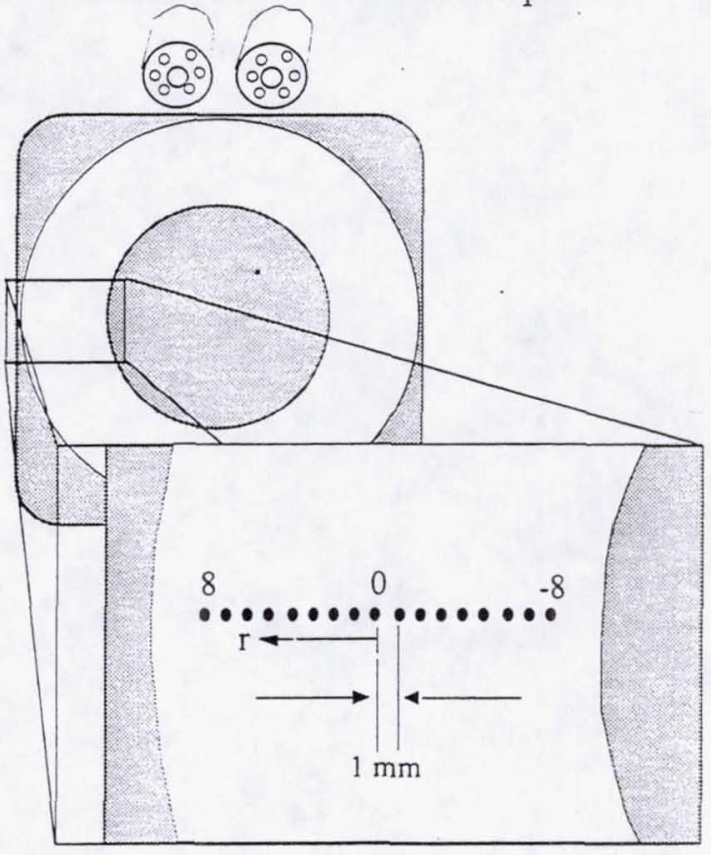

Fig. 6 Measurement Locations for Axial and Radial Velocity Determination

The laser was scanned over the fluorescence excitation spectrum at a rate of $0.002 \mathrm{~Hz}$ using a 3 $\mathrm{s}$ lock-in-amplifier time constant. Several additional scans were taken at a scan rate of $0.0002 \mathrm{~Hz}$ using a $30 \mathrm{~s}$ time constant. These longer scans improved the signal to noise substantially and were performed when high fidelity line shape data were desired. Some of the scans were repeated.

\section{Results and Discussion}

\section{Circumferential Velocity}

The circumferential velocity component was the first to be investigated. Two fluorescence excitation spectra, measured with the laser propagating in opposite directions with respect to the plume, are shown in Fig. 7. The laser was oriented such that it was collinear to the azimuthal velocity component.

Because the two measurements were taken at the same spatial location, in the center of the annular channel at the exit of the discharge chamber, the shift in velocity is twice the shift relative to a stationary source. The frequency centered between these two scans is $11976.72 \mathrm{~cm}^{-1}$. Energy levels published by Moore ${ }^{11}$ obtained from emission spectra indicate an unshifted frequency of $11976.64 \mathrm{~cm}-1$. The experimentally determined 
Page intentionally left blank 
unshifted line position was used through out this investigation as the unshifted frequency.

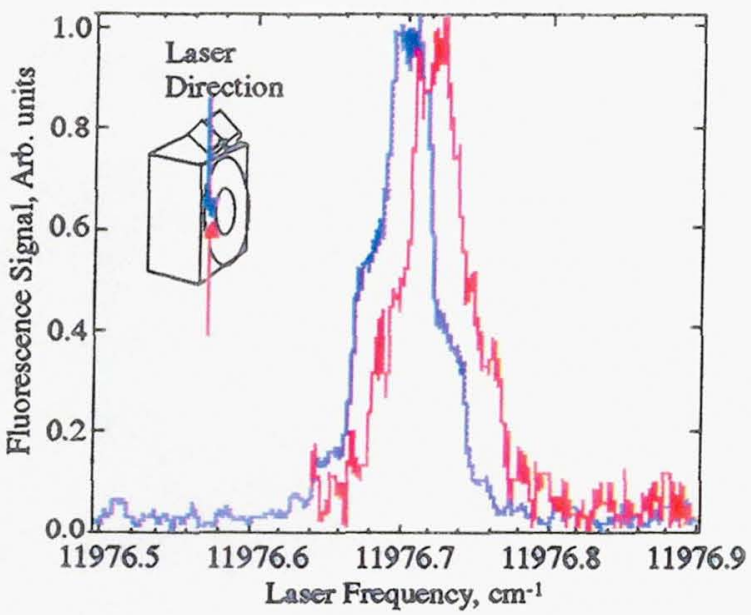

Fig. 7 Fluorescence Excitation Spectra Showing Circumferential Velocity Shift

The measured shift corresponds to a circumferential velocity of approximately 250 $\mathrm{m} / \mathrm{s}$ in the clockwise direction (looking towards the thruster). This direction of ion motion is consistent with the outward direction of the radial magnetic field. There was a $5 \times 10^{-3} \mathrm{~N}-\mathrm{cm}$ torque produced by the thruster assuming the thruster efflux was entirely composed of singly ionized xenon moving with this circumferential velocity. This corresponds to an equivalent force acting at a distance of $3.9 \mathrm{~cm}$ (the distance from the center of the thruster to the center of the annular discharge chamber) of approximately $2 \%$ of the measured thrust.

This estimate should serve as an upper bound because the plasma is not entirely composed of singly ionized xenon and the radial magnetic field is likely near its peak value at the measurement location. However, even this simplified assessment of torque is consistent with values published by Kozubsky ${ }^{12}$ who indicated the equivalent force was between $0.5 \%$ and $2 \%$ of the thrust. Other investigators have published higher estimates. Barnhart ${ }^{13}$ indicates a $1 \times 10^{-2}$ $\mathrm{N}-\mathrm{cm}$ torque, or $3 \%$ of the thrust andVaughn ${ }^{14}$ suggests a torque as high as $8 \%$ of the thrust. These values suggest circumferential velocities somewhat larger than measured for singly ionized xenon.

\section{Radial Velocity}

The radial velocities shown in Fig. 8 were measured $11 \mathrm{~mm}$ downstream of the discharge chamber exit plane $(1 \mathrm{~mm}$ downstream of the cathode). Each point was determined based on a curve fit of the measured fluorescence signal frequency variation. The $0 \mathrm{~mm}$ radial position denoted in Fig. 6 corresponds to the center of the annular channel. Coordinates in this case are positive going outward from the geometric center of the thruster (refer to Fig. 6). The variation of velocity with position indicates the necessity of high spatial resolution for accurate determination of radial velocity.

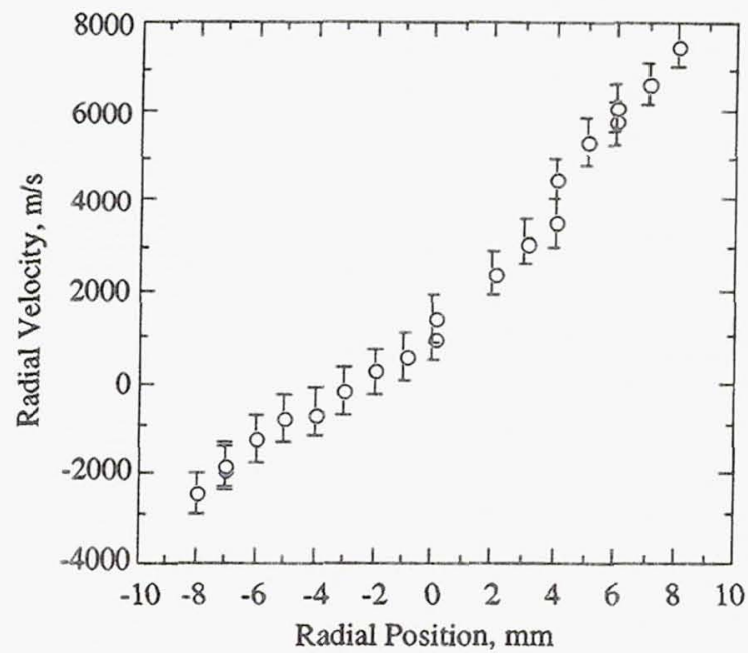

Fig. 8 Radial Velocity Measured $11 \mathrm{~mm}$ Downstream of the Discharge Chamber Exit Plane.

The flow was divergent with a near linear variation in radial velocity with radial distance. The position of zero radial velocity was determined to be several millimeters inward from the center of the channel. A radial velocity of $7500 \mathrm{~m} / \mathrm{s}$ was measured at a location $8 \mathrm{~mm}$ from the center of the channel. This was the largest radial velocity measured. Measurements were not made at larger radial distances, but the linear variation suggests that larger values of radial velocity may exist at larger radial distances. The magnitude of these radial velocities indicate that there are performance losses due to divergence in the exhaust for the SPT-100 operating at the single operating point tested.

Axial Velocity

Axial velocities were determined in conjunction with the radial velocity at the same measurement positions. The Doppler shift was measured with the laser sixty degrees from the axial direction in the same plane as the radial measurements. Because the laser was not coincident with axial ion velocity, the shift was 
Page intentionally left blank 
due to the axial and radial velocities. The axial velocity contribution to the shift was determined by removing the radial velocity's contribution. Fewer axial velocity measurements were taken due to time constraints. These data are shown in Fig. 9.

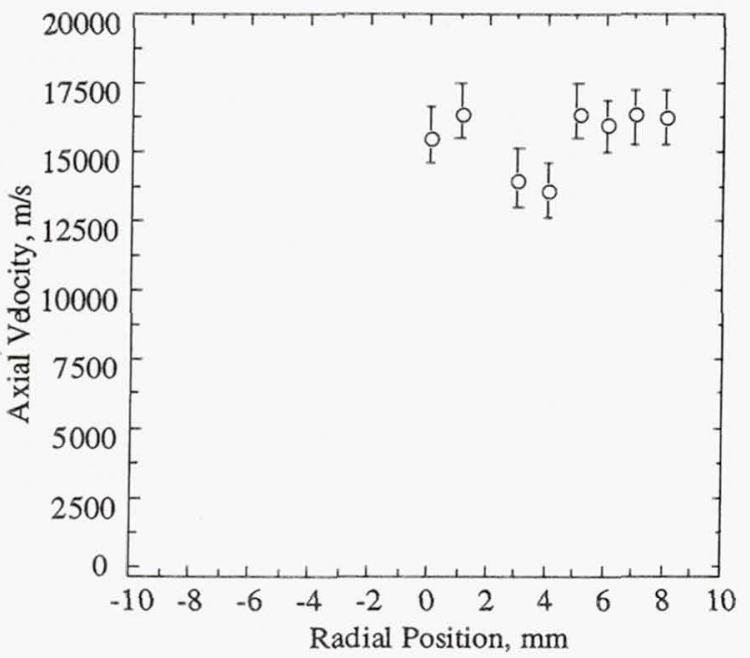

Fig. 9 Axial Velocity Measured $11 \mathrm{~mm}$ Downstream of the Discharge Chamber Exit Plane.

The magnitude of the axial velocities were consistent with a mass average velocity of approximately $15000 \mathrm{~m} / \mathrm{s}$ determined based on the measured specific impulse. The variation in axial velocity with discharge voltage was investigated by measuring the Doppler shift at the center of the channel while the thruster was operating at 250,300, and 350V. Assuming there was no change in the direction of the velocity vector with discharge voltage the axial velocity increased from $12700 \mathrm{~m} / \mathrm{s}$ at a discharge voltage of $250 \mathrm{~V}$ to $15600 \mathrm{~m} / \mathrm{s}$ at $300 \mathrm{~V}$ to $15800 \mathrm{~m} / \mathrm{s}$ at 350 $\mathrm{V}$. These values of the velocity varied less with the discharge voltage than the measured thrust reported by Brophy et. al. ${ }^{15}$

The axial and radial velocity measurements were used to construct velocity vectors. These vectors are depicted in Fig. 10. From this, the divergence of high energy ions was apparent. Data of this type may be of use for evaluating integration of such thrusters onto spacecraft because these high energy ions could cause significant sputter damage if they were to come in contact with a surface. Additional data of this type may allow a determination of zones of influence without relying solely on costly, time consuming direct impingement tests.

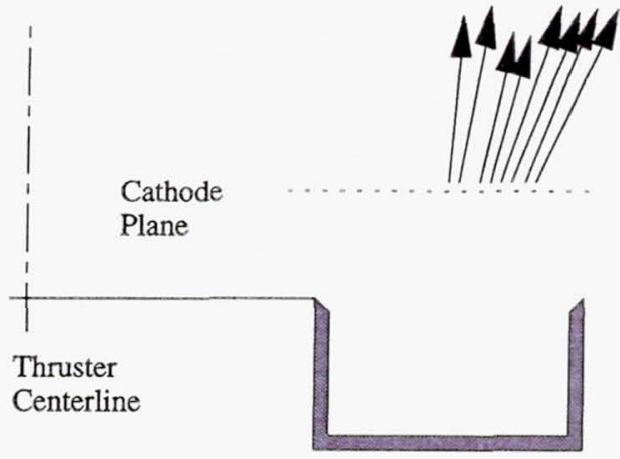

Fig. 10 Velocity Vectors $11 \mathrm{~mm}$ Downstream of the Discharge Chamber Exit Plane.

\section{Plasma Parameters}

The shape of the fluorescence excitation spectrum was evaluated to assess additional plasma parameters. Saturation behavior of the fluoresence transition was investigated and several non-saturated line shape measurements were recorded. Fig. 11 shows a measured line shape and a curve fit based on the previously discussed model. The scan was taken during an axial velocity determination. Each of the 20 individual transitions included in this curvefit were Doppler broadened an amount corresponding to $20,000 \mathrm{~K}$. Published fine structure constants were used for the $6 p$ state. ${ }^{10}$ Values for $5 d$ state where unavailable and were estimated based on the measured line shape. The temperature corresponding to the line shape measured in the azimuthal velocity determination was on the order of $800 \mathrm{~K}$. This is easily seen by comparing the scan widths shown in Figs. 7 and 11.

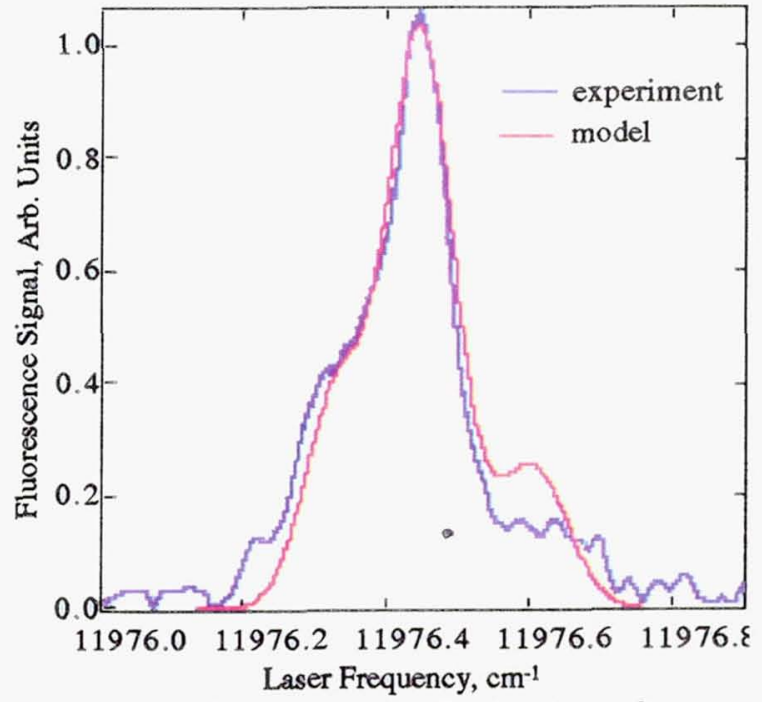

Fig. 11 Fluorescence Excitation Spectrum Measured During an Axial Velocity Determination and Analytic Fit. 
Page intentionally left blank 
The temperature used in the model to descibe the line width reflects a velocity spread in the laser direction. The experimental data indicate that the spread of velocity depended on direction. The spread in circumferential velocity was attributed to the ion's thermal motion because no circumferential potential gradients were expected. Therefore, the $800 \mathrm{~K}$ temperature used to model the scan taken in this direction was interpreted as the ion's kinetic temperature, a temperature near that of the discharge chamber walls. The comparatively large temperature needed to model the axial line shape indicated a spread in velocity that may be attributed to a variation in accelerating voltage for the ions. If the exhausting ions were created in a region of space with a potential difference there would be a spread in axial velocity corresponding to this potential difference. The velocity spread indicated by the scan shown in Fig. 11 corresponds to an approximately $3.4 \mathrm{eV}$ variation in ion energy (twice the $20000 \mathrm{~K}$ measured at 60 degrees from the axial direction). The largest velocity determined irrespective of direction was nearly $18000 \mathrm{~m} / \mathrm{s}$ corresponding to an ion energy of approximately $220 \mathrm{eV}$ which is large with regard to an $3.4 \mathrm{eV}$ energy spread.

\section{Conclusions}

A non-intrusive, laser induced fluorescence diagnostic was used to measure the velocity field for singly charged xenon in the exhaust of a SPT. Axial, radial, and circumferential velocities were measured and velocity vectors were reconstructed from these data. These data demonstrate that detailed information with high spatial resolution can be obtained which can be used for performance assessment and spacecraft integration of these thrusters.

Momentum torques caused by ion trajectories with a circumferential velocity component were estimated. Based on measured circumferential velocities, this torque was conservatively estimated to be $5 \times 10^{-2} \mathrm{~N}-\mathrm{cm}$. Radial velocity measurements indicate a relatively divergent flow field. Axial velocities consistent with the measured thrust were determined. Ion velocity vectors in the exhaust of the SPT were reconstructed from the measurements of the individual velocity components. The magnitude of the ion velocity vector indicates that ion energies as high as $220 \mathrm{eV}$ are present in the exhaust with an approximately $3.5 \mathrm{eV}$ energy spread. This spread in energy was determined based on the measured fluorescence excitation spectrum and an analytic model constructed to simulate this transition.

\section{References}

1. Sankovic, J.M., Hamley, J.A., and Haag, T.W., "Performance Evaluation of the Russian SPT Thruster at NASA LeRC," IEPC-93-094, Sept. 1993.

2 Hamley, J.A., Hill, G.M., and Sankovic, J.M., "Power Electronics Development for the SPT-100 Thruster," IEPC-93-044, Sept. 1993.

3. Pencil, E.J., "Far-Field Plume Sputtering Characterization of the Stationary Plasma Thruster (SPT-100)," IEPC-93-098, Sept. 1993.

4 Myers, R.M. and Manzella, D.H., "Stationary Plasma Thruster Plume Characteristics," IEPC-93096, Sept. 1993.

5 Manzella, D.H., "Stationary Plasma Thruster Plume Emissions," IEPC-93-097, Sept. 1993.

6. Liebeskind, J.G., "Temperature and Velocity Diagnostic for an Arcjet Plume Using LaserInduced Fluorescence," PhD Thesis, Stanford University, April 1994.

7. Erwin, D.A., Pham-Van-Diep, G.C., Deininger; "Laser Induced Fluorescence Measurements of Flow Velocity in an Arcjet Using LIF," AIAA Journal, Vol.29, No.8, August 1991.

8. Ruyten, W.M. and Keefer, D., "Laser Fluorescence Velocimetry of an Arcjet Exhaust Plume,"IEPC-91-093, October 1991.

9. CRC Handbook of Chemistry and Physics, 60th edition Weast, R.C. and Astle, M.J. eds., CRC Press, Boca Raton, FL, 1979.

10. Alvarez, E., et. al., "Isotope Shift and Hyperfine Structure Measurements in XeII in a Laser Beam Experiment," Physica Scripta, Vol. 20, 1979.

11. Moore, C.E.,"Atomic Energy Levels, Volume III, United States National Bureau of Standards Circular 467, 1958.

12. Kozubsky, K., Zhurin, V., and Higham, J., "Disturbance Torques Generated by the Stationary Plasma Thruster," AIAA-93-2394, June 1993.

13. Barnhart, D., "EPOCH: Electric Propulsion Operational Characterization Experiment," AIAA92-3196, July 1992.

14. Vaughn, C.E., "An Updated Assessment of Electric Propulsion Technology for Near-Earth Space Missions," AIAA-92-3202.

15. Brophy, J.R., Barnett, J.W., Sankovic, J.M., and Barnhart, D.A., "Performance of the Stationary Plasma Thruster: SPT-100," AIAA-92-3155, July 1992. 
Public reporting burden for this collection of information is estimated to average 1 hour per response, including the time for reviewing instructions, searching existing data sources, gathering and maintaining the data needed, and completing and reviewing the collection of information. Send comments regarding this burden estimate or any other aspect of this collection of information, including suggestions for reducing this burden, to Washington Headquarters Services, Directorate for Information Operations and Reports, 1215 Jefferson Davis Highway, Suite 1204, Arlington, VA 22202-4302, and to the Office of Management and Budget, Paperwork Reduction Project (0704-0188), Washington, DC 20503.
1. AGENCY USE ONLY (Leave blank)
2. REPORT DATE
August 1994
3. REPORT TYPE AND DATES COVERED
Final Contractor Report

4. TITLE AND SUBTITLE

Stationary Plasma Thruster Ion Velocity Distribution

6. AUTHOR(S)

$06-42-31$

C-NAS3-27186

David H. Manzella

7. PERFORMING ORGANIZATION NAME(S) AND ADDRESS(ES)

NYMA, Inc.

Engineering Services Division

2001 Aerospace Parkway

5. FUNDING NUMBERS

Brook Park, Ohio 44142

8. PERFORMING ORGANIZATION

REPORT NUMBER

E-9094

9. SPONSORING/MONITORING AGENCY NAME(S) AND ADDRESS(ES)

10. SPONSORING/MONITORING AGENCY REPORT NUMBER

National Aeronautics and Space Administration

Lewis Research Center

Cleveland, Ohio 44135-3191

NASA CR-195379

AIAA-94-3141

11. SUPPLEMENTARY NOTES

Prepared for the 30th Joint Propulsion Conference cosponsored by AIAA, ASME, SAE, and ASEE, Indianapolis, Indiana, June 27-29, 1994. Project Manager, Paul Choma, Space Propulsion Technology Division, NASA Lewis Research Center, organization code 5330, (216) 433-5750.

\begin{tabular}{l|l} 
12a. DISTRIBUTION/AVAILABILITY STATEMENT & 12b. DISTRIBUTION CODE
\end{tabular}

Unclassified - Unlimited

Subject Category 20

13. ABSTRACT (Maximum 200 words)

A non-intrusive velocity diagnostic based on laser induced fluorescence of the $5 d^{4} F_{5 / 2}-6 p{ }^{4} D_{5 / 2}$ singly ionized xenon transition was used to interrogate the exhaust of a $1.5 \mathrm{~kW}$ Stationary Plasma Thruster (SPT). A detailed map of plume velocity vectors was obtained using a simplified, cost-effective, non-intrusive, semiconductor laser based scheme. Circumferential velocities on the order of $250 \mathrm{~m} / \mathrm{s}$ were measured which implied induced momentum torques of approximately $5 \times 10^{-2} \mathrm{~N}-\mathrm{cm}$. Axial and radial velocities were evaluated one mm downstream of the cathode at several locations across the width of the annular acceleration channel. Radial velocities varied linearly with radial distance. A maximum radial velocity of $7500 \mathrm{~m} / \mathrm{s}$ was measured $8 \mathrm{~mm}$ from the center of the channel. Axial velocities as large as $16,500 \mathrm{~m} / \mathrm{s}$ were measured.

\section{SUBJECT TERMS}

Stationary plasma thruster; Laser induced fluorescence; Xenon

15. NUMBER OF PAGES

13

16. PRICE CODE

$\mathrm{A03}$

17. SECURITY CLASSIFICATION OF REPORT

Unclassified

18. SECURITY CLASSIFICATION OF THIS PAGE

Unclassified

19. SECURITY CLASSIFICATION OF ABSTRACT

Unclassified 